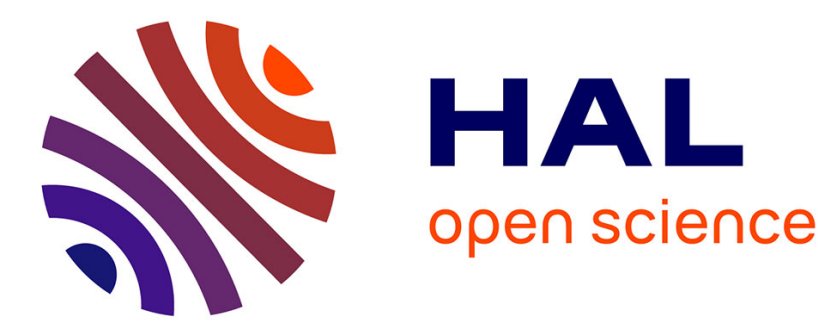

\title{
Un indice de mesure du collaboratif client-fournisseur
}

Carole Donada, François Lenglet, Caroline Mothe, Jean-Jacques Nillès

\section{To cite this version:}

Carole Donada, François Lenglet, Caroline Mothe, Jean-Jacques Nillès. Un indice de mesure du collaboratif client-fournisseur. Revue Française de Gestion, 2015, 41 (251), pp.69-85. 10.3166/RFG.251.6985. hal-01301383

\section{HAL Id: hal-01301383 \\ https://hal.univ-grenoble-alpes.fr/hal-01301383}

Submitted on 12 Apr 2016

HAL is a multi-disciplinary open access archive for the deposit and dissemination of scientific research documents, whether they are published or not. The documents may come from teaching and research institutions in France or abroad, or from public or private research centers.
L'archive ouverte pluridisciplinaire HAL, est destinée au dépôt et à la diffusion de documents scientifiques de niveau recherche, publiés ou non, émanant des établissements d'enseignement et de recherche français ou étrangers, des laboratoires publics ou privés. 


\title{
Un indice de mesure du collaboratif client-fournisseur
}

\author{
Carole Donada $^{\mathrm{a}}$, François Lenglet ${ }^{\mathrm{b}}$, Caroline Mothe ${ }^{\mathrm{c}}$, Jean-Jacques Nillès ${ }^{\mathrm{d} 1}$
}

\section{Résumé}

Cet article présente un outil de mesure du collaboratif dans les relations clientfournisseur : le PEAK Collaborative Index ${ }^{\circledR}$ (PCI). Fondé sur les bases théoriques des normes transactionnelles collaboratives et des approches philosophiques sur les vertus cardinales de justice, de courage, de prudence et de tempérance, le PCI a été testé auprès de près de 300 entreprises engagées dans des relations collaboratives. Il révèle l'importance de vertus propédeutiques comme le respect et la courtoisie des personnes mais aussi des comportements inter-organisationnels non-opportunistes ancrés dans la recherche de justice et la générosité.

Mots-clés : collaboratif, indice, modèle de mesure, normes collaboratives, vertus

\footnotetext{
${ }^{\text {a }}$ Professeur, ESSEC Business School

${ }^{\text {b }}$ Maître de Conférences en Sciences de Gestion, IREGE, Université de Savoie

${ }^{\text {c }}$ Professeur des Universités, IREGE, Université de Savoie

d Maître de Conférences en Sciences de Gestion, IREGE, Université de Savoie

${ }^{1}$ Les travaux présentés dans le présent article sont issus du programme de recherche du projet PEAK (Purchasing European Alliance for Knowledge) destiné à développer les relations collaboratives clients fournisseurs. Les auteurs remercient Thésame, les entreprises adhérentes et les financeurs institutionnels du projet PEAK : le Fonds pour l'Innovation et l'Industrie (F2I), l'UDIMERA, le Conseil Régional Rhône-Alpes, le Conseil Général de la Haute-Savoie et le Pôle de compétitivité Mont-Blanc Industries.
} 


\begin{abstract}
This paper presents a collaborative measurement tool in the customer-supplier relationships: the PEAK (C) Collaborative Index (PCI). Based on the theoretical foundations of relational norms and philosophical approaches of the cardinal virtues of justice, courage, prudence and temperance, the PCI has been tested for 3 years with hundreds of firms engaged in collaborative relationships. It reveals the importance of virtues such as respect and courtesy but also of non-opportunistic inter-organizational behaviors rooted in the search for justice and generosity.
\end{abstract}

Keywords: collaborative, index, measurement model, relational norms, virtues 


\section{Introduction}

L'expression 'relation collaborative' est à la mode. Académiques, experts professionnels et praticiens vantent les qualités de ce mode de gouvernance relationnel, théoriquement capable de combler les imperfections du marché tout en levant les contraintes des structures hiérarchisées. Dans l'ensemble des relations collaboratives possibles, les échanges économiques entre des entreprises clientes et leurs fournisseurs font l'objet d'une attention particulière car ils sont au cœur des stratégies d’outsourcing, des processus conjoints de création d'innovations ou des politiques de responsabilité sociale des entreprises à l'égard de leurs parties prenantes. Mais, qu'entend-on réellement par relation collaborative clientfournisseur? Comment interpréter les dires d'un acheteur qui prétend avoir des relations collaboratives avec ses fournisseurs ? Y a-t-il une mesure de la collaboration qui permette de comparer les perceptions des entreprises clientes avec celles des fournisseurs ? Telles sont les questions à l’origine de cette recherche exploratoire.

Les cadres explicatifs des collaborations inter-organisationnelles et de leurs effets sur la performance générale des entreprises reposent généralement sur une combinaison de théories. Leurs auteurs invoquent tantôt des arguments économiques (l'économie des coûts de transaction et ses extensions), des arguments socio-politiques (théorie du pouvoir et de la dépendance, théorie des contrats), des arguments issus des sciences de l’organisation (théorie des normes contractuelles ou des réseaux), ou encore des arguments d'ordre sociopsychologique (théorie des relations personnelles, sciences comportementales, etc.). Les recherches sur les relations collaboratives empruntent donc les concepts de ces différents cadres théoriques et les combinent pour formuler des modèles de mesure plus ou moins sophistiqués. A l'exception de quelques travaux publiés dans les revues académiques spécialisées dans l'éthique des affaires, nous avons observé que le cadre théorique de la philosophie n’est quasiment jamais mobilisé. Pourtant, les propositions de la philosophie sur 
les vertus cardinales de justice, de prudence, de courage et de tempérance sont a priori à même de donner du sens à ce qu'on entend par collaboration. Nous avons également observé que les modèles empiriques de mesure du collaboratif étaient extrêmement variés et de composition très inégale. Ceux destinés aux tests d’hypothèses théoriques sont souvent réduits à un nombre restreint d'items et ceux élaborés par les professionnels pour évaluer la qualité des relations avec leurs partenaires ne sont généralement pas construits sur des bases théoriques solides et ne considèrent souvent qu'une seule partie des échanges (le point de vue du client ou du fournisseur).

Notre objectif est de proposer un instrument de mesure du collaboratif client-fournisseur qui soit 1) théoriquement solide, 2) statistiquement valide et fiable et 3) construit pour devenir un indice récurrent de mesure du collaboratif client-fournisseur. L'indice que nous proposons (appelé le PCI ou PEAK Collaborative Index) répond à ces exigences. Il trouve ses racines dans les théories des organisations sur les normes contractuelles et dans les approches philosophiques sur les vertus cardinales. Cet outil s'appuie sur 19 items qui se regroupent en deux variables latentes représentant deux conditions sans lesquelles il ne peut y avoir de collaboratif, à savoir le respect des personnes et le non-opportunisme organisationnel qui se caractérise par le sens de la justice (traduite par l’honnêteté, l’intégrité ou l'entièreté) et la générosité (la disposition au don ou libéralité).

Nous avons construit le PCI en 5 étapes : (1) une revue de la littérature théorique sur les collaborations inter-organisationnelles, (2) une revue des modèles empiriques, (3) la construction conceptuelle et empirique de l'indice, (4) l'administration d'un questionnaire pour récolter les données et (5) l’analyse de la validité et de la fiabilité de l’indice. Ces étapes sont décrites et synthétisées dans les trois parties qui composent cet article. Nous commençons par une synthèse des différentes littératures mobilisées avant de décrire les 
fondements conceptuels et empiriques du PCI. La troisième partie présente les résultats de l’analyse statistique ainsi que les contributions managériales de cet instrument de mesure.

\section{Revue de la littérature : vertus et normes collaboratives}

La collaboration inter-organisationnelle repose sur une logique partenariale caractérisée par « des relations stratégiques intentionnelles entre des entreprises indépendantes qui partagent des objectifs compatibles, luttent pour des bénéfices mutuels et reconnaissent un niveau élevé d'interdépendance mutuelle. Elles unissent leur effort pour atteindre les objectifs que chaque entreprise, agissant seule, ne pourrait atteindre facilement » (Mohr et Spekman, 1994, p. 135). Les collaborations entre des entreprises clientes et leurs fournisseurs ont fait l'objet de nombreuses études reprises dans des revues de littérature détaillées et des méta-analyses statistiques (Donada et Nogatchewsky, 2005a). Ces travaux confirment les gains liés à une démarche collaborative sur les achats et les approvisionnements, les avantages en termes d'efficacité des processus et de flexibilité, les nouvelles possibilités de combinaison des ressources pour un rendement combiné supérieur aux rendements individuels additionnés, l’amélioration des niveaux de qualité des offres et une capacité d'innovation accrue.

\section{Les cadres théoriques}

Les cadres théoriques mobilisés dans les différents travaux sont variés et complémentaires. Les auteurs empruntent aux économistes des coûts de transaction les concepts d’opportunisme, d’actifs spécifiques ou de fréquence des relations pour évaluer la qualité transactionnelle des échanges entre des clients et des fournisseurs. Ils mobilisent la théorie du pouvoir et de la dépendance et l'approche par les ressources et les compétences pour évaluer les jeux de pouvoir entre les partenaires, leurs complémentarités sur les ressources clefs et par là même leurs interdépendances. Les théories des réseaux d’entreprises sont mobilisées pour aborder les problèmes de gouvernance et de structures organisationnelles. Enfin, la littérature 
en psychosociologie offre des clefs pour comprendre les mécanismes comportementaux des partenaires. Les concepts issus de ces différentes approches sont combinés dans des modèles théoriques pour décrire et expliquer les relations client-fournisseur. L’analyse de ces modèles révèle que les chercheurs font tous implicitement ou explicitement référence à ce que les tenants de l'approche relationnelle ${ }^{2}$ appellent « normes contractuelles ».

Ces normes correspondent à des comportements attendus et partagés, au moins partiellement, par les parties prenantes d'une relation. Ce sont des mécanismes informels de gouvernance qui font office de contrat social (Macneil, 1980 ; Hatten et al., 2012). Les normes contractuelles sont des facteurs décisifs dans la création d'une atmosphère favorable à la collaboration (Blois et Ivens., 2006). Elles réduisent les risques d'opportunisme (Tangpong et al., 2009) et elles fournissent aux participants une indication sur le degré de confiance et d'assurance dans l'échange et vis-à-vis de l'avenir (Ott et Ivens, 2009). Les normes facilitent l'engagement et la création de valeur et, finalement, elles influencent positivement la performance (Heide et John, 1992 ; Ott et Ivens, 2009). La synthèse des résultats de toutes les études traitant de ces sujets peut se résumer de la façon suivante : les normes contractuelles facilitent la coopération entre les individus et leurs organisations parce qu'elles reposent sur des comportements qui réduisent l'opportunisme et créent une harmonie favorable à la pérennité des échanges (Ott et Ivens, 2009).

Macneil (1980) met en évidence dix normes «contractuelles » (cf. Tableau 1), qui sont subdivisées en deux groupes: cinq normes relationnelles (intégrité du rôle, solidarité, flexibilité, bien-fondé des moyens, normes supra-contractuelles) et cinq normes transactionnelles (réciprocité, mise en œuvre du planning, réalisation des promesses, réparation, confiance et attentes, création et restriction du pouvoir) :

\footnotetext{
2 Cette approche s’est développée dans la lignée des propositions théoriques de Macneil (1980) sur les contrats sociaux, celles de l'IMP Group sur les échanges inter-organisationnels (Håkansson et al., 1982), de Morgan et Hunt (1994) sur la confiance et l'engagement ou de Dyer et Singh (1998) sur les rentes relationnelles pour identifier les normes contractuelles (ou collaboratives) indispensables à une collaboration inter-organisationnelle.
} 


\begin{tabular}{|l|l|}
\hline \multicolumn{1}{|c|}{ Normes Relationnelles } & \multicolumn{1}{|c|}{ Normes Transactionnelles } \\
\hline $\begin{array}{l}\text { 1. Intégrité du rôle } \\
\text { 2. Solidarité }\end{array}$ & 1. Réciprocité \\
3. Flexibilité & 2. Mise en œuvre du planning \\
$\begin{array}{l}\text { 4. Bien-fondé des moyens } \\
\text { 5. Normes supra-contractuelles } \\
\text { (intensification de la norme } \\
\text { d’harmonisation avec la matrice sociale) }\end{array}$ & 4. Réparation, confiance et attentes \\
\hline
\end{tabular}

Tableau 1 : Les normes contractuelles de Macneil (1980)

Nous appellerons ces normes « collaboratives » pour garder la cohérence avec notre objet de recherche. En outre, le terme «contractuel» peut prêter à confusion car, dans son sens courant, il renvoie au contrat - alors que, chez Macneil, son acception est plus large.

\section{Les cadres empiriques}

Les cadres empiriques des modèles d'analyse des collaborations client-fournisseur sont de deux types. Le premier est celui des cadres élaborés par les chercheurs pour tester des propositions théoriques et qui visent avant tout la qualité scientifique. Les articles de recherche qui présentent ces cadres développent toujours leurs apports managériaux, mais rares sont ceux qui produisent des outils de référence pour les gestionnaires de supply chains. Deux contributions font exceptions à nos remarques. La première est celle de Simatupang et Sridharan (2005) qui proposent un outil de mesure du collaboratif entre fournisseurs et distributeurs à partir de 25 items : le Collaboration Index. Cet outil est solidement ancré dans les propositions théoriques de la gestion des opérations et il permet d'obtenir un score de collaboration statistiquement valide et fiable sur l'importance du partage de l'information, de la synchronisation des décisions et de l'alignement des incitations. La seconde contribution est celle de Henke et al. (2008) avec le Working Relations Index ${ }^{T M}$ (WRI). Largement déployé dans l'industrie automobile, le WRI quantifie le niveau et les effets de l'expérience des 
fournisseurs et leur confiance vis-à-vis de leurs clients. C’est un outil de mesure de la qualité des relations fournisseurs à partir de cinq dimensions et dix-sept items ${ }^{3}$. L'ancrage théorique du WRI est celui de l'approche relationnelle, même si cet outil ne traite que partiellement des normes collaboratives.

Le second type de cadres empiriques des collaborations client-fournisseur est celui élaboré par les cabinets de conseil. Ces modèles visent avant tout la praticité managériale ; ils sont construits pour aider les entreprises au pilotage de leurs collaborations client-fournisseur. Ils ne s’appuient pas vraiment sur des fondements théoriques solides et leur qualité statistique est difficilement évaluable par des analystes extérieurs.

De notre revue de la littérature émergent trois constats. Premièrement, les cadres théoriques mobilisés par les chercheurs qui étudient les collaborations client-fournisseur sont hétérogènes. Ces cadres sont combinés pour former des modèles, alors même qu’ils reposent sur des fondements épistémologiques différents. Un cadre intégrateur s’appuyant sur les normes collaboratives (dans la lignée de l'article fondateur de MacNeil, 1980) semble pertinent. Le deuxième constat est que les travaux académiques empiriques sur le collaboratif ont des portées managériales trop restreintes pour devenir des 'outils référents’ d'un pilotage de relations collaboratives client-fournisseur. Même les modèles les plus pertinents comme le CI ou le WRI ne comblent pas entièrement ces limites. Le CI peut être aisé à mettre en place dans une organisation, mais il réduit la mesure du collaboratif à l'analyse de variables très opérationnelles sur la qualité d'une chaîne d'approvisionnement. Le WRI est plus riche sur les dimensions relationnelles, mais il ne permet pas de croiser les perceptions des partenaires et ne mesure donc pas la qualité d'une collaboration inter-organisationnelle. Enfin, si les outils de mesure développés par les cabinets de conseil ont des qualités opérationnelles indéniables, ils ne s’appuient sur aucun fondement théorique solide.

\footnotetext{
${ }^{3}$ Pour plus de détails sur le WRI, cf. http://www.ppi1.com/services/working-relations-index/
} 
Forts de ces constats, nous avons décidé de développer un indice de mesure du collaboratif client-fournisseur, le PEAK Collaborative Index ${ }^{\odot}$ (PCI) qui s’inscrive dans un cadre théorique cohérent et solide, qui soit robuste statistiquement et qui soit utile pour des professionnels en charge des relations collaboratives dans les organisations.

\section{Cadre conceptuel et structure du PCI}

\section{Cadre conceptuel}

Les comportements organisationnels associés aux normes dans les relations client-fournisseur sont idiosyncratiques. Les états de l'art des recherches sur ces comportements confirment cette hétérogénéité (Ivens et Blois, 2004 ; Blois et Ivens, 2006 ; Kulmann, 2012 : Donada et Nogatchewsky, 2005b) en mettant en avant la diversité des normes : la coercition restreinte, la communication, la compréhension mutuelle, la confiance, la coopération, la coordination, l’engagement, la flexibilité, l’harmonisation, l’intégrité de rôle, la longévité, la mise en œuvre conjointe du planning, les normes supra-contractuelles, le partage d'information, la réciprocité, la résolution harmonieuse des conflits, la solidarité ou la transparence. Bien que chaque auteur puisse justifier ses choix pour telle ou telle norme selon ses références, le panorama des possibilités est tellement éclaté qu'il est difficilement appropriable dans une démarche de validation théorique (Blois et Ivens, 2006, 2007).

C’est pourquoi il nous a semblé indispensable d'ancrer notre outil de mesure du collaboratif client-fournisseur dans un cadre théorique suffisamment large pour intégrer les normes collaboratives, mais aussi suffisamment structurant pour les restreindre à quelques dimensions cardinales. Compte-tenu de ces éléments, la littérature philosophique sur les vertus nous est apparue pertinente car peut-il exister une collaboration entre deux partenaires sans les vertus cardinales de justice, d'altruisme (ou respect), de courage, de prudence et de tempérance ? 
Les premiers écrits au sujet des vertus sont l'œuvre des philosophes grecs. Dans « La République », Platon (427-348 avant Jésus-Christ) distingue quatre vertus principales : la sagesse (Livre IV, 428b-429a), le courage (Livre IV, 429a-430c), la tempérance (Livre IV, 430d-432b) et la justice (Livre IV, 432b-444a). Platon considère les vertus comme des attitudes fermes, des dispositions stables, des perfections habituelles de l'intelligence et de la volonté qui règlent les actes, ordonnent les passions et guident la conduite. Elles procurent facilité, maîtrise et joie. Aristote (384-322 avant Jésus-Christ) dans l’ « Ethique à Nicomaque » utilise une distinction différente. « Nous distinguons, en effet, les vertus intellectuelles et les vertus morales : la sagesse, l’intelligence, la prudence sont des vertus intellectuelles ; la libéralité et la modération sont des vertus morales » (Livre I, chapitre 13). Il met à part la justice qu'il considère comme la plus haute des vertus. Un peu plus loin, il précise la signification de la vertu : " Ainsi donc, la vertu est une disposition à agir d’une façon délibérée consistant en un juste milieu relative à nous, laquelle est rationnellement déterminée et comme la déterminerait l’homme prudent » (Livre II, chapitre 6).

Les points communs entre ces définitions sont que les vertus sont des dispositions à agir, qu'elles sont acquises et qu'elles visent l'action positive pour l'individu et pour la cité. La vertu se définit ainsi comme la propriété spécifique d'une chose ou d'un être, qui en constitue l'excellence propre, une "disposition permanente à produire certains effets, une perfection qualitative définie et spéciale" (Robin, 1947, p. 72). C’est "...une qualité humaine acquise dont la possession et l'exercice tendent à permettre l'accomplissement des biens internes aux pratiques et dont le manque rend impossible cet accomplissement" (MacIntyre 1997, p. 186).

La tradition philosophique retient quatre vertus dites cardinales, dans le sens où elles sont les pivots de l'action humaine (latin cardo: charnière, pivot) et où, par conséquent, elles déterminent les autres vertus. La justice nous dit ce qui est juste et ce que l'on a le droit de faire en vue du bien d'autrui. Le courage nous donne la volonté de réaliser ce qui est bien, et 
d'agir avec détermination. La prudence nous fait voir, dans chaque situation, par quels moyens on peut y parvenir. La tempérance enfin, permet d'agir dans l'équilibre et en adaptant notre action à la réalité. L’époque moderne y ajoute l'altruisme comme respect pour les intérêts fondamentaux de l'autre (qui était inclut dans la justice pour Platon, mais chez qui il a du coup un caractère moins central).

Dans le cadre de relations inter-organisationnelles, la justice donne à chaque partenaire ce qui d'après le droit positif (la loi) ou naturel (la morale) lui revient. Elle détermine le caractère légal ou équitable des relations. Elle assure le respect des engagements explicites ou implicites des partenaires. Le courage donne aux partenaires la volonté de réaliser ce qu'il est bien de faire. Il permet de s’engager, de décider et donc de prendre des responsabilités. Le courage détermine aussi leurs capacités à affronter l'échec, à surmonter les difficultés et à se dépasser. La prudence c’est la sagesse des partenaires dans l'action, l'éthique de leurs responsabilités. Elle leur fait voir quel est le bien commun, et par quels moyens ils peuvent y parvenir. La prudence est fondée sur les connaissances et la recherche de la vérité. Enfin, la tempérance permet d'agir dans l'équilibre, en s'adaptant à la réalité et au contexte d'interaction, de modérer les désirs des partenaires et de maîtriser leurs impulsions pour préserver ou créer des conditions relationnelles favorables à long terme.

Cardinale ou non, une vertu a toujours une fonction particulière mais elle est présente dans les autres, car chacune a besoin des autres. Une vertu séparée deviendrait vice. Par exemple, le courage sans la prudence est témérité ; sans la tempérance, il est impulsion non maîtrisée (Aristote, EN, II, 7). De même, chaque vertu est la vertu, mais aucune n'est toute la vertu et c'est de leur combinaison que les dispositions à agir se forment. Pour illustrer ce point, la justice et le courage appellent l’honnêteté ou l'intégrité des partenaires ; la prudence et à la tempérance renvoient à leur solidarité, leur générosité ou leur libéralité etc. 
Enfin, pour se déployer dans des comportements collaboratifs, les vertus cardinales ont besoin des vertus propédeutiques sans lesquelles les interactions sociales ne peuvent se réaliser. Il s’agit notamment des vertus de politesse, de courtoisie et de respect des autres. L’idée simple de cette propédeutique est qu’il faut toujours «commencer par dire bonjour avant de demander quelque chose ».

\section{Structure du PCI}

Le PCI a pour objectif de mesurer de manière stable la qualité d'une relation collaborative entre des entreprises clientes et fournisseurs. Les fondements théoriques de ces dimensions sont issus des travaux sur les normes relationnelles et les vertus. Ces vertus sont une adaptation des quatre vertus cardinales de Platon (justice, courage, prudence et tempérance), une cinquième ayant été rajoutée : l'altruisme (ou respect de l'autre). Nous l'avons retenu en raison de son importance dans la réflexion éthique contemporaine et de son caractère central dans la relation commerciale. Ces cinq vertus se combinent entre elles pour caractériser des comportements d’intégrité, de générosité, d’engagement, de responsabilité, de libéralité ou de sagesse. Ces comportements sont en adéquation avec les normes d'engagement, de communication, de continuité, de flexibilité, d'intégrité de rôle, de solidarité ou de transparence (Macneil, 1980), qui sont au cœur des collaborations inter-organisationnelles. Sachant qu'une vertu séparée devient vice, nous formulons l’hypothèse que les indicateurs des vertus d’une collaboration doivent être fortement liés entre eux.

L’Annexe 1 propose une opérationnalisation des 5 vertus cardinales en 19 items basée sur la revue de littérature. Compte-tenu de la littérature sur les normes et sur les vertus, nous émettons l'hypothèse que le PCI recouvre un indice uni-factoriel.

\section{Données et méthode de traitement}


La collecte des données a été réalisée entre avril et mai 2014. Le questionnaire a été diffusé en format électronique via Internet à un échantillon d'entreprises ou de professionnels appartenant à divers réseaux partenaires de PEAK, par qui l’e-mailing a été transmis. La base de données de certains de ces partenaires étant confidentielle (souhait de Thésame ${ }^{4}$, qui pilote le programme PEAK), nous n’avons pas pu avoir une connaissance précise du nombre de contacts. Le nombre de réponses enregistrées a été de 349. Les caractéristiques de l’échantillon sont les suivantes :

- Statut des répondants (position dans la relation) : clients $40 \%$; fournisseurs $60 \%$.

- Taille des entreprises : grandes entreprises (plus de 5000 salariés) : 16\% ; ETI (entre 250 et 4999 salariés) : 24\% ; PME (entre 11 et 249 salariés) : 37\% ; TPE (moins de 10 salariés) : 23\%.

- Secteurs d’activité : industrie : 56\% ; services : 22\% ; énergie : 9\% ; distribution : 8\% ; transport et logistique : 5\%.

Pour le traitement des données, la première étape a consisté à purifier l’instrument de mesure en menant une série d’analyses factorielles exploratoire sous SPSS sur les 19 items générés. La solution factorielle est ensuite validée par modélisation structurelle. Les analyses confirmatoires sont menées sous Amos avec la méthode du maximum de vraisemblance (ML). Elles sont établies sur la base de données réduite à $\mathrm{n}=279$ après élimination des données manquantes : l'absence de données manquantes est une condition requise par ce logiciel pour permettre l'ajustement de fonctions. Cette taille d'échantillon est conforme aux prérequis de la méthode du maximum de vraisemblance et ne justifie pas de recourir à la méthode des moindres carrés partiels (PLS), plus adaptée aux petits échantillons (n <200)

\footnotetext{
4 Thésame est un réseau technologique rhône-alpin pour les entreprises en mécatronique, productique et management de l'innovation (http://www.thesame-innovation.com/).
} 


\section{Le PCI, un indice de mesure du collaboratif client-fournisseur}

\section{Résultats}

La procédure d'épuration par analyses factorielles itératives conduit à éliminer successivement les items mal représentés selon les critères classiques (communautés ou pourcentage de variance restituée dans la solution factorielle, analyse des poids factoriels, des corrélations item-ensemble ; voir Parasuraman et alii (1990)). Finalement, une solution à 9 items émerge, la qualité de représentation minimale s’établit à 0,514 (cf. Tableau 2). Le test d'adéquation des variables manifestes avec l'analyse factorielle est positif, (valeur de l'indice KMO : 0,909). Le critère de Kaiser (valeurs propres supérieures à 1), tout comme le test de pente de Cattell conduisent à retenir deux facteurs, lesquels restituent 61,6\% de la variance des items. Après rotation oblique, la corrélation inter-facteurs s’établit à 0,462. La fiabilité du premier facteur est très bonne $(\alpha=0,89) .^{5}$

\begin{tabular}{|c|c|c|c|}
\hline \multirow{2}{*}{ Items retenus } & \multirow{2}{*}{$\begin{array}{l}\text { Qualité de la } \\
\text { représentation }\end{array}$} & \multicolumn{2}{|c|}{ Scores factoriels } \\
\hline & & Facteur 1 & Facteur 2 \\
\hline Courtoisie & ,684 & & 0,795 \\
\hline Retenue & ,702 & & 0,838 \\
\hline Equilibre de la relation & ,514 & 0,714 & \\
\hline Progrès partagé & ,735 & 0,894 & \\
\hline Honnêteté intellectuelle & ,629 & 0,785 & \\
\hline Consistance & ,636 & 0,649 & \\
\hline Solidarite & ,605 & 0,642 & \\
\hline Développement compétences & ,688 & 0,902 & \\
\hline Transparence & ,558 & 0,621 & \\
\hline Fiabilite $(\alpha)$ & & 0,889 & \\
\hline \% Variance restituée & & 51,524 & 10,094 \\
\hline Corrél. inter-facteurs & & \multicolumn{2}{|c|}{0,462} \\
\hline
\end{tabular}

Tableau 2 : Analyse factorielle exploratoire de l'indice PCI

\footnotetext{
${ }^{5}$ Le calcul de l'alpha de Cronbach est très sensible au nombre d'items, et n'a donc guère de sens pour le second facteur (il vaut ici 0,606). On peut toutefois apprécier sa cohérence interne par l'examen de la corrélation entre les deux items, qui s’établit ici à 0,456 ( $<<0,001)$.
} 
L’interprétation des axes permet de conclure que le premier facteur correspond aux vertus cardinales, tandis que le second illustre les vertus propédeutiques.

L'analyse factorielle confirmatoire révèle un bon ajustement du modèle aux données : les paramètres estimés sont tous significatifs ( $<<0,001$ ) (cf. Tableau 3) et les indices d'ajustements (cf. Tableau 4) sont tous conformes aux seuils habituellement retenus en modélisation par équations structurelles (e.g. Roussel et al., 2002). La corrélation interfacteurs est de 0,727 .

\begin{tabular}{|l|c|}
\hline Items (facteur) & Poids factoriel \\
\hline Courtoisie (F2) & 0,715 \\
\hline Retenue (F2) & 0,650 \\
\hline Equilibre de la relation (F1) & 0,649 \\
\hline Progrès partagé (F1) & 0,757 \\
\hline Honnêteté intellectuelle (F1) & 0,753 \\
\hline Consistance (F1) & 0,776 \\
\hline Solidarité (F1) & 0,747 \\
\hline Développement compétences (F1) & 0,702 \\
\hline Transparence (F1) & 0,720 \\
\hline
\end{tabular}

Tableau 3 : Paramètres de l’analyse factorielle confirmatoire

\begin{tabular}{|l|l|l|l|}
\hline Khi-deux $: 38,295$ & Ddl $: 25$ & Khi-deux/ddl $: 1,532$ & P $: 0,043$ \\
\hline GFI $: 0,971$ & AGFI $: 0,947$ & \\
\hline CFI $: 0,980$ & RMSEA $: 0,043$ & \\
\hline
\end{tabular}

Tableau 4 : Ajustement du modèle de mesure du PCI

La validité de trait est également confirmée dans ses deux composantes, la validité convergente et la validité discriminante. Le calcul du rhô de validité convergente de Fornell et Larcker (1981) permet de vérifier que la variable latente correspondant au facteur 1 partage

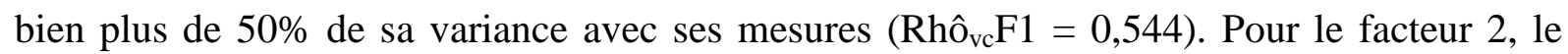
rhô $_{\mathrm{vc}}$ atteint pratiquement ce seuil $(0,492)$ alors même qu'il est pénalisé par le faible nombre d’items. Pour s'assurer de la validité discriminante, il convient de comparer deux modèles de mesure : dans le premier, les facteurs se corrèlent librement, tandis que dans le second la corrélation est forcée à 1 (Bagozzi et Yi, 1989). S’agissant de modèles nichés (même nombre de variables, une seule contrainte additionnelle), il est possible de comparer leur ajustement 
par un test de différence de $\chi^{2}$. Le critère de validité discriminante impose alors que le modèle à corrélations libres présente un meilleur ajustement, apprécié par un plus faible $\chi^{2}$, que le modèle à corrélation fixée à 1 . Le $\chi^{2}$ du modèle contraint vaut 116,306 , et la différence de $\chi^{2}$ $(78,011)$ entre les modèles est significative $(\mathrm{p}<0,001)$. Ces résultats confirment la supériorité du modèle bidimensionnel et établissent la validité discriminante. Ainsi, les deux variables latentes théoriquement différentes (les vertus cardinales et les vertus propédeutiques) sont également distinctes au niveau statistique (F1 et F2).

\section{Discussion des résultats}

La vertu étant une disposition à agir, c'est dans les actions ou les comportements qu'elle se réalise. Aucune vertu n'étant naturelle, il faut donc devenir vertueux. Et c'est en les faisant, comme expliquait Aristote, que nous les apprenons. Lorsqu'il s'agit de déterminer le comportement juste dans un contexte d'action déterminé, chaque vertu peut être déclinée en comportements typiques qui l'expriment. C’est ce qui a été réalisé en traduisant les vertus cardinales dans les comportements qui s’expriment dans le PCI.

Le premier facteur rassemble toutes les vertus indispensables au collaboratif (les «vertus cardinales ») parce qu’elles empêchent l’opportunisme ${ }^{6}$ de se développer. On y retrouve les vertus cardinales de la justice, de la prudence, du courage et de la tempérance présentes dans leurs déclinaisons telles que l’intégrité, la générosité, la sagesse, la tolérance, l’humanité, l’altruisme ou la libéralité. La qualité de la représentation statistique de toutes ces variables sur l'axe factoriel montre qu’il n’y a pas de vertu isolée. Cela fait écho à la théorie qui nous rappelle que chaque vertu est complémentaire des autres parce qu’une vertu séparée deviendrait vice. Par exemple, le courage sans la prudence est témérité ; sans la tempérance, il est impulsion.

\footnotetext{
${ }^{6}$ L’opportunisme est défini comme une attitude consistant à régler sa conduite selon les circonstances du moment, que l'on cherche à utiliser toujours au mieux de ses intérêts.
} 
Ces variables correspondent aux vertus cardinales, c'est à dire à la mise à disposition de ressources et de compétences pour faire grandir l'autre, pour donner pour aider, développer la relation et, peut-être, un jour, recevoir. On est ici dans la lignée des idées sur le don et le contredon développées par Marcel Mauss, fondateur de l’ethnologie scientifique française et auteur du célèbre Essai sur le don (1925) - et récemment reprises par Caillé et Grésy (2014). Sans bonne volonté, rien ne se fait. Mais elle ne se décrète pas. Ainsi, sans don, il n’est pas de bonne volonté et d'efficience possibles. Ces notions intègrent des vertus d’intégrité, de justice, de générosité et de solidarité. L’intégrité diminue l’incertitude des relations, la justice va au-delà des principes de loi en intégrant les éléments liés à l’équité, la générosité fait qu’on agit au-delà de tout texte selon Descartes, et la solidarité est le principe de toutes les vertus selon Platon.

Le second facteur couvre les dimensions liées aux vertus propédeutiques du collaboratif, qui sont en quelque sorte des «pré-vertus » de la collaboration. Ce sont des attitudes personnelles (ou liées à la culture d'entreprise et à son influence sur les attitudes individuelles) qui renvoient à des qualités nécessaires comme la politesse, la courtoisie, le respect des autres, l'empathie ou la retenue. Ce sont des attitudes nécessaires mais elles sont cependant insuffisantes. Elles donnent les prémices, le « faire naître » du collaboratif, pour paraphraser Comte-Sponville sur la politesse, « ce n'est qu’un commencement mais c'en est un », et il est nécessaire.

\section{Conclusion}

L’enquête PEAK PCI 2014 présente des résultats très intéressants pour les entreprises en termes de management de la relation collaborative clients/fournisseurs. En effet, sur un échantillon varié et couvrant les différentes populations de l’industrie française (entreprises de toutes tailles et tous secteurs d'activité), les statistiques confirment que l'index de la 
collaboration est composé de deux dimensions sous-jacentes principales. La première traduit le respect et les vertus propédeutiques. La seconde est relative à la gestion de la relation collaborative, ou aux normes et vertus cardinales qui prévalent au sein de cette relation. Il s’agit donc d'une part de traits fondamentaux de la personnalité (comme le sens du respect, de la politesse) qui déterminent le comportement des interlocuteurs, d'autre part de la présence de normes collaboratives qui facilitent la relation inter-organisationnelles (aide au développement de compétences, solidarité, consistance des décisions, honnêteté, équilibre de la relation, progrès partagé, transparence, etc.). Ces résultats sont autant confirmés pour les entreprises clientes et les fournisseurs.

Le cadre théorique des normes transactionnelles collaboratives, qui s’appuie sur l'approche philosophique par les vertus, est à ce jour celui qui nous semble le plus complet pour appréhender le collaboratif client-fournisseur et nous proposons d'en présenter les principales variables dans la section suivante. Toutefois, nous ajoutons à ce cadre celui des théories philosophiques sur les vertus. Les propositions de la philosophie sur les vertus cardinales comme la justice, la prudence, le courage ou la tempérance sont parfaitement à même de donner du sens à ce qu'on entend par collaboration car ce sont des « habitus » de la volonté qui habilitent l’homme à agir bien.

\section{Contributions managériales}

Le modèle de mesure du PEAK PCI tel qu'il a été élaboré est un instrument essentiel dans le management des relations collaboratives entre clients et fournisseurs et, plus largement, de la supply chain. En proposant quelques axes simples de gestion de cette relation sur lesquels il est aisé pour les entreprises de s’appuyer, toute entreprise pourra s’engager dans la voie du progrès tant ces normes collaboratives sont importantes pour le succès de la relation - et pour que chaque partie puisse en tirer les bénéfices escomptés dans une relation collaborative 
équilibrée, durable et responsable. Aussi convient-il non seulement d'aligner les incitations dans ce sens, afin de valoriser la prise en compte de ces normes collaboratives par les acteurs, mais aussi de recruter les «bonnes personnes » pour manager ces relations, c’est à dire celles qui sont sensibles aux vertus relationnelles et comportementales pour le succès d'une relation coopérative.

\section{Limites et pistes de recherches futures}

Cette étude exploratoire constitue la première étape d'un programme de recherche sur l'état des relations collaboratives entre clients et fournisseurs dans la mesure où l'index crée sera désormais évalué chaque année. Au niveau théorique, la difficulté de l’exercice tient, comme pour toute recherche portant sur les phénomènes inter-organisationnels, à l'articulation des niveaux individuel et organisationnel pour le développement des comportements propices aux relations collaboratives. Nous avons ici pris le parti, par rapport aux recherches et autres indices mélangeant ces deux niveaux, de nous focaliser sur le niveau individuel et sur les comportements puisque, in fine, ce sont bien les individus sur lesquels repose le succès des relations client-fournisseur, et des relations inter-organisationnelles de manière générale.

De futures recherches pourraient tenter d'articuler ces normes relationnelles individuelles, reposant sur des vertus et des comportements individuels, avec des «supra-normes » au niveau organisationnel (liées à la culture et aux valeurs de l'entreprise). Ce lien avec la culture d'entreprise et avec le management par les valeurs, qui sont au cœur de la RSE (responsabilité sociale d'entreprise) et notamment de l'ISO 26000, pourrait être creusé dans des recherches ultérieures. De même il semble pertinent à l'avenir d’insérer le PCI dans un réseau nomologique pour évaluer l'impact des normes collaboratives sur des indicateurs de performance économique mais aussi sur des pratiques d'innovation ou de RSE. Dans le même ordre d'idées mais de manière plus pragmatique, il conviendrait de porter un regard renouvelé 
sur les programmes de formation (tant initiale que continue) pour promouvoir ces comportements collaboratifs. Il reste beaucoup à faire pour parvenir à modifier les comportements dans les relations industrielles, mais le récent intérêt pour l’index PCI de la part de certaines filières françaises laisse présager que les entreprises, tout comme les individus, sont de plus en plus sensibles à ce changement de mentalités et de modes relationnels, tant au niveau individuel (Caillé et Grésy, 2014) qu’organisationnel. Ce changement peut aussi être vu comme un retour aux sources philosophiques. En effet, les organisations qui fonctionnent bien savent reconnaître dans le cycle du don et dans ceux qui s’y adonnent la véritable source de la coopération efficace, de la confiance et du travail pris à cœur. Le mauvais gestionnaire, qui s’acharne à tout contrôler et rationaliser, tue la « poule aux œufs d'or ». En enfermant tout le monde dans le cercle vicieux du chacun pour soi, comme le dit La Fontaine, il « perd tout en voulant tout gagner ».

\section{Bibliographie}

Aristote (350 av. JC). Ethique à Nicomaque (traduit par J. Tricot, Paris, Vrin, coll. Bibliothèque des Textes Philosophiques - Poche, 1990).

Bagozzi R.P. et Yi Y., (1989). « On the Use of Structural Equation Models in Experimental Designs », Journal of Marketing Research, Vol. 26, n³, p. 271-285.

Blois K. J. et Ivens B. S. (2006). « Measuring relational norms: some methodological issues ", European Journal of Marketing, vol. 40, n³/4, p. 352-365.

Blois K.J. et Ivens B.S. (2007). « Method issues in the measurement of relational norms », Journal of Business Research, vol. 60, n5, p. 556-565.

Caillé A. et Grésy J-E. (2014). La Révolution du don. Le management repensé à la lumière de l'anthropologie, Seuil, Paris.

Calvi R., Le Dain M.A. et Harbi S. (2000). « Le pilotage des partenariats client-fournisseur dans l'industrie », Revue française de gestion industrielle, vol. 19, n¹, p. 5-15.

Comte-Sponville A. (1995). Petit traité des grandes vertus, PUF, Coll. Points, Paris. 
De Cannière M.H., De Pelsmacker P. et Geuens, M. (2009). «Relationship quality and the theory of planned behavior models of behavioral intentions and purchase behavior ». Journal of Business Research, vol. 62, n 1, p. 82-92.

Donada C. et Nogatchewsky G. (2005a). «Dépendance asymétrique dans les alliances verticales : Comment un client vassal contrôle-t-il ses fournisseurs ?», Management International, vol. $10, \mathrm{n}^{\circ} 1$, p. 63-74.

Donada C. et Nogatchewsky G. (2005b). « Vingt ans de recherches empiriques en marketing sur la performance des relations client-fournisseur?", Recherche et Applications en Marketing, vol. 20, $\mathrm{n}^{\circ}$ 4, p. 71-96.

Fynes B., de Búrca S. et Mangan J. (2008). « The effect of relationship characteristics on relationship quality and performance ». International Journal of Production Economics, vol. 111, $\mathrm{n}^{\circ} 1$, p. 56-69.

Hatten K.J., James W.L., Fink R.C. et Keeler J.P. (2012). « Macneil's Relational Norms and His Non-mirrored Ends Propositions », Journal of Marketing Channels, vol. 19, n 1, p. 116.

Heide J.B. et John G. (1992), « Do Norms Matter in Marketing Relationships? », Journal of Marketing, vol. 56, $\mathrm{n}^{\circ}$ 2, p. 32-44.

Henke J.W., Parameswaran R. et Pisharodi M. (2008). « Manufacturer price reduction pressure and supplier relations », Journal of Business \& Industrial Marketing, vol. 23, $\mathrm{n}^{\circ}$ 5, p. 287-300.

Ivens B.S. et Blois K.J. (2004). « Relational exchange norms in marketing: A critical review of Macneil’s contribution », Marketing theory, vol. 4, n³, p. 239-263.

Jap S.D., Manolis C. et Weitz B.A. (1999). «Relationship quality and buyer-seller interactions in channels of distribution ». Journal of Business Research, vol. 46, n 3, p. 303-313.

Grégoire Y. et Fisher R. J. (2006). «The effects of relationship quality on customer retaliation ». Marketing Letters, vol. 17 n¹, p. 31-46.

Lahiri S. et Kedia, B.L. (2009). « The effects of internal resources and partnership quality on firm performance: An examination of Indian BPO providers ». Journal of International Management, vol. 15, n 2, p. 209-224.

Lee J.N. et Kim Y.G. (1999). «Effect of partnership quality on IS outsourcing success: conceptual framework and empirical validation ». Journal of Management information systems, vol. $15, \mathrm{n}^{\circ} 4$, p. 29-61.

Macintyre, A. (1997). Après la vertu, PUF, Paris. 
Macneil I.R. (1980). The New Social Contract: An Inquiry into Modern Contractual Relations, Yale University Press, New Haven, CT.

Mohr J. et Spekman, R. (1994). "Characteristics of partnership success: partnership attributes, communication behavior, and conflict resolution techniques ", Strategic Management Journal, vol. 15, $\mathrm{n}^{\circ}$ 2, p. 135-152.

Ott C. et Ivens B.S. (2009). «Revisiting the Norm Concept in Relational Governance », Industrial Marketing Management, vol. 38, n 6, p. 577-583.

Parasuraman A., Zeithaml V.A. et Berry L. (1990). «SERVQUAL: une échelle multi-items de mesure des perceptions de la qualité de service par les consommateurs », Recherche et Applications en Marketing, Vol. 5, n¹, p. 19-42.

Platon (380 av. JC). La République (traduit par G. Leroux, Flammarion, Paris, 2002).

Robin L. (1947). La morale antique. Presses universitaires de France, Paris.

Roussel P., Durrieu F., Campoy E. et El Akremi A. (2002), Méthodes d'équations structurelles : recherche et applications en gestion, Economica, Paris.

Simatupang T.M. et Sridharan, R. (2002). « The Collaborative Supply Chain », International Journal of Logistics Management, vol. 13, n 1, p. 15-30.

Tangpong C., Hung K.T. et Ro Y.K. (2009). « The interaction effect of relational norms and agent cooperativeness on opportunism in buyer-supplier relationships ", Journal of Operations Management, vol. 28, n 5, p. 398-414. 
Annexe 1 - Opérationnalisation des 5 vertus cardinales

\begin{tabular}{|c|c|c|c|}
\hline Vertu & Item & Libellé & Sources \\
\hline \multicolumn{4}{|l|}{ Respect } \\
\hline 1 & $\begin{array}{l}\text { Reconnaissance } \\
\text { positive }\end{array}$ & $\begin{array}{l}\text { Percevoir dans les réalisations du partenaire leur aspect positif en } \\
\text { priorité et l'exprimer }\end{array}$ & Grégoire et Fisher, 2006 \\
\hline 2 & Courtoisie & $\begin{array}{l}\text { Marquer la considération que l'on a pour l'autre par ses attitudes, } \\
\text { comportements et paroles }\end{array}$ & Lee et Kim, 1999 \\
\hline 3 & Solidarité & $\begin{array}{l}\text { Dans les difficultés ou dysfonctionnements éventuels, avoir une attitude } \\
\text { de soutien pour son partenaire }\end{array}$ & Lee et Kim, 1999 ; Fynes et al., 2008 ; Kang 2013 \\
\hline 4 & $\begin{array}{l}\text { Développement } \\
\text { compétences }\end{array}$ & $\begin{array}{l}\text { Lorsque ses compétences sont supérieures à celles du partenaire dans un } \\
\text { domaine donné, chercher à le faire progresser }\end{array}$ & Fynes et al., 2008 \\
\hline \multicolumn{4}{|l|}{ Tempérance } \\
\hline 1 & Disponibilité & $\begin{array}{l}\text { Consacrer au partenaire le temps et l'écoute dont il a besoin pour réaliser } \\
\text { sa mission }\end{array}$ & Lee et Kim, 1999 \\
\hline 2 & Retenue & $\begin{array}{l}\text { Faire preuve de pondération et de contrôle de soi dans ses } \\
\text { comportements, ses propos ou ses décisions }\end{array}$ & Platon \\
\hline 3 & $\begin{array}{l}\text { Equilibre de la } \\
\text { relation }\end{array}$ & $\begin{array}{l}\text { Poursuivre ses objectifs sans que ce soit au détriment des intérêts du } \\
\text { partenaire et au contraire en cherchant à les préserver }\end{array}$ & Jap et al., 1999 ; Lee et Kim, 2000 \\
\hline 4 & Consistance & $\begin{array}{l}\text { Prendre ses décisions en les articulant à la stratégie de son organisation } \\
\text { et non pas de façon opportuniste }\end{array}$ & Lahiri et Kedia, 1999 \\
\hline \multicolumn{4}{|l|}{ Justice } \\
\hline 1 & Intégrité & Agir conformément au droit et aux principes communs & Fynes et al., 2008 ; De Cannière et al., 2000 \\
\hline 2 & Concurrence loyale & $\begin{array}{l}\text { Respecter les règles d'une concurrence loyale fondée uniquement sur } \\
\text { des critères objectifs de qualité ou de performance }\end{array}$ & Calvi et al., 2000 \\
\hline 3 & Fiabilité & Respecter les engagements formels ou informels que l'on a pris. & Jap et al., 1999 ; Fynes et al., 2008 ; Lahiri et Kedia, 1999 \\
\hline 4 & Progrès partagé & $\begin{array}{l}\text { Lorsque les projets conduits en commun font grandir son entreprise, en } \\
\text { faire profiter le partenaire }\end{array}$ & Lee et Kim, 1999 ; Fynes et al., 2008 \\
\hline \multicolumn{4}{|l|}{ Prudence } \\
\hline 1 & Justification & $\begin{array}{l}\text { Fonder ses décisions sur une argumentation et la partager pour chercher } \\
\text { à les faire comprendre }\end{array}$ & Lee et Kim, 1999 \\
\hline 2 & Objectivité & Fonder ses décisions ou appréciations sur des faits précis & Calvi et al., 2000 \\
\hline 3 & $\begin{array}{l}\text { Niveau } \\
\text { interlocuteurs }\end{array}$ & $\begin{array}{l}\text { Allouer les ressources humaines utiles et nécessaires à l’atteinte des } \\
\text { objectifs fixés ensemble }\end{array}$ & Lahiri et Kedia, 1999 \\
\hline 4 & Horizon & $\begin{array}{l}\text { Gérer la relation et les projets communs dans une perspective de long } \\
\text { terme }\end{array}$ & Jap et al., 1999 ; Grégoire et Fisher, 2006 ; Fynes et al., 2008 \\
\hline \multicolumn{4}{|l|}{ Courage } \\
\hline 1 & $\begin{array}{l}\text { Honnêteté } \\
\text { intellectuelle }\end{array}$ & Chercher la cohérence entre son discours, sa pensée et ses actes & Lee et Kim, 1999 ; Grégoire et Fisher, 2006 \\
\hline 2 & Transparence & Communiquer de façon transparente avec son partenaire & Lee et Kim, 1999 ; Jap et al., 1999 ; Fynes et al., 2008 \\
\hline 3 & Proactivité & $\begin{array}{l}\text { Prendre des initiatives pour maintenir et améliorer la qualité de la } \\
\text { relation }\end{array}$ & Jap et al., 1999; Kang 2013 \\
\hline
\end{tabular}

